\title{
Reflections on the Research Methods Used in an Investigation of Cross-modal Collaborative Information Seeking
}

\author{
Dena Al Thani and Tony Stockman \\ School of Electronic Engineering and Computer Science \\ Queen Mary University of London \\ London, UK \\ d.al-thani,t.stockman@qmul.ac.uk
}

\begin{abstract}
Evaluating collaborative information seeking can be challenging, often more than individual information seeking. This can be attributed to the complexity of the interaction as the study involves a number of users using the systems. While this is particularly important, but rather hard when the users employ different modalities accessing the systems. In this paper, we describe a semi-structured combination of qualitative and quantitative methods used to investigate visually impaired and sighted web users' collaborative information seeking behavior. We critically reflect on the benefits and limitations of the methods used, and discuss practical considerations in application of such methods in a cross-modal setting.
\end{abstract}

Keywords-research methods; evaluation; collaborative information seeking; cross-modal interaction

\section{INTRODUCTION}

Evaluating a collaborative information seeking (CIS) environment can be very challenging, owing to its complex nature which involves multiple users and a variety of interactions [1]. The evaluation of CIS systems is rarely discussed in research papers, and still less evaluating a CIS system that is used by people who employ different interaction modalities such as audio or haptics. The majority of usability studies of computer systems have been carried out with people that employ the visual modality as the main means of interaction. Thus, usability evaluation methods that have evolved over the years are designed almost entirely with only these users in mind.

The interest in inclusive design [2][3] has been gradually growing in research, especially in the last two decades. The introduction of concepts such as 'inclusive design' and 'universal usability' and the development of W3C work in the field of accessibility have brought some attention to the inclusion of non-standard populations in the design and evaluation process. These approaches emphasize the role of potential users in the design and evaluation of interfaces. Most designers are barely aware that individuals with physical, sensory or cognitive disabilities often fall into a system's target user group and the majority of usability studies of computer systems have been carried out with able-bodied users [4]. with increasing emphasis being placed on inclusion and universal usability, there is must be a growing trend to include users with disabilities in usability studies during formative and summative design processes, and to assess conformance with disabilityrelated regulations. In this paper we reflect on the research methods used in our work when evaluating a cross-modal collaborative information Seeking (CCIS) system where visually impaired (VI) and sighted users collaboratively search the web together. We here use the term "cross-modal" to refer to a situation where different users employ different interaction modes to engage with an application. Specifically, in the case of our study, sighted users employed a standard visual interface, while VI users employed speech-based screenreaders to access the system. In this research project, the goal was to understand the process of CCIS and examine how the knowledge gained could be used to inform the design of a system to support CCIS. The research work included a series of studies reported in [5], we started by conducting an onlinesurvey, the results of which showed that a significant amount of cross-modal collaborative search goes on. An exploratory observation study was conducted to investigate the challenges faced and behaviour patterns that occur when people performed CCIS. This study included 14 pairs of VI and sighted users in which participants employed their tools of choice, that is their web browser, note taker and preferred communications system. The study examined how concepts from the "mainstream" (CIS) literature played out in the context of cross-modal interaction. Following this, we surveyed mainstream CIS systems to select the most eligible software package that had adequate levels of accessibility and satisfied the design recommendations from the initial study. Having identified that one of the surveyed systems was, with some enhancements, relatively accessible, we developed Jaws [6] script ${ }^{1}$ and employed other Jaws features to improve its accessibility.

We then performed a second study, using the same participants undertaking search tasks of a similar complexity as before, but this time using the CIS system with its accessibility enhanced by the Jaws scripts. The aim of this study was to

\footnotetext{
${ }^{1}$ Jaws scripts are a programmatic means of extending the functionality of the Jaws screen-reader system to handle accessibility issues not rendered adequately by the standard Jaws application.
} 
explore the impact on the CCIS process of introducing a mainstream CIS system, enhanced for accessibility. In this paper we reflect on the research methods used in these studies, discussing their benefits and challenges. We start by providing an overview of the overall approach taken before going on to reflect on the individual research methods employed in each of the phases of the project outlined above.

\section{OVERVIEW OF APPROACH TAKEN}

User studies are an empirical method commonly used in HCI to understand users' needs and behaviour, and evaluate a situation in which technology is used. In such studies, quantitative methods are frequently employed to collect and analyse data in numerical form. The analysis of this data is often related to hypothesis testing and provides a basis for comparison between groups and/or between interactions performed under different conditions [7]. Qualitative methods have also long played an essential role in these types of studies [8]. They are increasingly being employed in the field of HCI as they provide holistic views of situations and therefore support the researcher in perceiving the issues and patterns which occur in evaluation data.

In our research we are exploring the interaction between VI and sighted users conducting collaborative web searches. This is the first study we are aware of in this area, and so it is by definition exploratory. We are interested in quantitative measures (for example in order to make comparisons between CCIS activities in different conditions), but we are also interested in the collaborative process and user experience, a full picture of which requires a combination of quantitative and qualitative measures. Therefore we conducted semi-structured qualitative studies that involve questionnaires, observational studies and interviews. Semi-structured qualitative studies mainly focus on developing an understanding of a situation in an exploratory way [9]. There is also some structure to the process of analysis that includes systematic coding of data, which is quantitative in nature. The method also considers quantitative data which is crucial to the analytical process. The user studies we designed collected both qualitative and quantitative data. Qualitative methods such as the structured observations and semi-structured interviews allowed us to understand the process of collaboration and the behaviour of users during the CIS process. Quantitative data, such as the duration of a given information seeking stage, query term length, number of search results explored and stored etc. represent essential measures to understand the process. This broad combination of data provides an in-depth view of CCIS activities performed under different conditions, and so provides a basis for understanding the process and developing a rationale for how to better support and enhance it.

\section{OUTLINE OF THE WORK DONE}

There follows an outline of the main stages of the work.

\section{A. Preliminary Survey}

We conducted a preliminary online questionnaire that captured information about VI and sighted individuals' personal search activities and information management habits.
It also investigated how often CCIS activities occur between VI and sighted web users. This initial survey helped us to identify how frequently CCIS activity occurs. It provided us with an insight into the nature of the process and so underpinned the experimental design of the first study. The survey showed that both VI and sighted respondents do regularly engage in CIS activities. The survey also shows that accessibility issues have an influence on the differences in CIS behaviour between VI and sighted users. In fact, VI respondents demonstrated an awareness of a range of web accessibility issues in their survey responses.

\section{B. Study 1}

We conducted an exploratory observational study that investigated the challenges faced and behaviour patterns that occur in CCIS activity between 14 pairs of VI and sighted users [10]. In this study, participants used their tools of choice, that is their preferred web browser, note taker and communications system. We observed behaviour patterns that occurred in CCIS activities in both co-located and distributed settings. The study explored issues such as awareness, division of labour and results management in the presence of cross-modality. The study also looked at how the different stages of information seeking were performed. This allowed us to investigate CCIS behaviour and the challenges that arose due to the cross-modal interaction, the tools used and how the process was affected by the accessibility of web sites visited to perform the tasks. The study allowed us to develop an understanding of different aspects of the CIS process in the context of cross-modal interaction, and the influence that cross-modal interaction has on the different stages of the process.

\section{The Follow-up to Study 1}

Following study 1, we undertook scenario-based interviews with seven VI and seven sighted users who were involved in study 1 . These interviews investigated their web search results management habits to deepen our understanding of the results of the exploratory study and to further explore the results management stage. The study provides an insight into how users retrieved information, the technologies used and the differences in the ways that VI and sighted users manage retrieved information. The outcomes from study 1 plus the data from these additional interviews yielded results which allowed us to form an understanding of the CCIS process when employing the users' tools of choice, without the use of any system specifically supporting the collaborative process. The understanding gained from this first observational study and the follow-on interviews enabled us to compile a set of design recommendations for CCIS systems features.

\section{Functionality and Accessibility Review}

The purpose of the next stage then became to identify candidate systems that might be used to explore these questions. There was of course no guarantee that any such system would exist. The investigation consisted of three stages; firstly, the available mainstream CIS systems were surveyed to choose the ones that were both available and suitable in their coverage of the CIS process. The features and functionalities of the chosen systems were then mapped to the design features 
from Study1. This was followed by a task-based accessibility assessment; the final choice of the system to be used was based on the outcome of this final stage. The outcome was that there was no single system which was sufficiently accessible as it stood. However there was one system that was close enough that sufficient improvements to its accessibility could be made within a suitable timescale in order for it to be employed in a study to address the above research questions. The next stage of the project involved developing and testing the required improvements to this system's accessibility. At the end of this process we had an extended tool which was sufficiently accessible for use in a study to address the research questions we wished to explore.

\section{E. Study 2}

Study 2 is an observational study that explores CCIS behaviour between VI and sighted users using the extended CIS tool in both co-located and distributed settings. This study was carried out with the same 14 pairs of participants that took part in study 1 . The reason for recruiting the same participants is solely related to the research questions in study 2 that investigate the effects of the interface on the different concepts of CIS and the IS behaviour of the participants. It is important to assert here, that the overall aim was to understand the changes in behaviour, process and challenges when a mainstream CIS tool with extended accessibility features was employed, and not to evaluate the design or the functionality of the interface. Areas of particular interest included performance, workspace awareness, information made available to collaborators, division of labour and, stages of the information seeking process.

Quantitative and qualitative data were collected and the findings analysed and compared with the results of study 1 . Using the results from study 1 as a baseline provided us with insights into the differences in collaborative behaviour patterns, and processes manifested under these different conditions. It highlighted the effects the interface had on individual information seeking behaviour, awareness and user interaction. It provided knowledge about how users employ interface features when performing CIS tasks. The extended CIS tool positively affected user experience and performance. The features available enabled users to put less effort into coordination and so lead to improved task performance. It also provides evidence that VI users benefited from the accessibility enhancements implemented, which demonstrates that a few yet well studied adjustments made through scripting and changes to the settings of the access tool, can enable users experience, engagement and performance to be positively and effectively enhanced. The final stage of the process was to produce a set of recommendations towards the inclusive design of a tool to support CCIS.

In studies 1 and 2, we followed the same within participant design. In the studies, each pair of participants performed the tasks under two conditions (co-located and distributed). One task was performed in a co-located setting and the other task was performed in a distributed setting. The main reason for choosing this approach was to lessen the impact of individual differences and to see how behaviour patterns and performance varied across conditions for each pair. It is important to ensure that the order in which participants perform tasks does not bias the results [7, p177]. To minimize order effects care was taken in the studies to counterbalance the order of the tasks. In each study, seven pairs worked in the co-located condition followed by the distributed condition; while the other seven pairs started with the distributed condition followed by the co-located condition.

\section{QUESTIONNIERS}

Questionnaires were used three times in our work. They were used as a method to collect data online about CCIS activities in the preliminary study as reported in the previous section. Part of the motivation for conducting the survey online was because online questionnaires have the potential to access groups that are typically under-represented in research [11]. A lot of information can be collected from a large number of people in a short period of time and in a relatively cost effective way. This initial questionnaire was divided into two major parts. The first part contained demographic and background-related questions (seven questions), and the second part investigated CIS behaviour (six questions). We also used questionnaires techniques in study 1 , to capture demographic information about the participants' search experience and proficiency, as well as the VI participants' use of access technology. In study 2, questionnaires were used post-study in order to collect data related to accessibility and usability satisfaction levels.

Despite having the advantage of reaching relatively large numbers of people from the target group in a short period of time, questionnaires are clearly less flexible and have a number of other disadvantages when compared to interviews for a number of reasons [12]. Firstly, people may read questions differently and this may in turn affect their responses and the subsequent analysis of results. This places an emphasis on careful design of the questionnaire. Another common issue is the accuracy and the truthfulness of the answers collected via the web [13].

The online survey we conducted allowed us to have an initial understanding of how often CIS takes place. The survey is an attempt to explore the current state and nature of CIS activity in both groups. It showed that members of both populations do regularly engage in CIS activities. This survey was not intended to be comprehensive, but the intention was to obtain an idea of the extent of CIS activity in the two populations, and to begin to examine differences in CIS behaviour between them.

\section{USER OBSERVATIONAL STUDY}

In studies 1 and 2, we observed users undertaking structured CIS tasks in pairs, with the pairs comprising one VI and one sighted participant. In structured observations, researchers direct the participant[s] to perform certain activities. In other words, the researcher sets the scene and then observes users' actions accordingly, as the objective is to investigate a specific behaviour [13]. The advantage of such studies is that they allow users to perform a task in more natural settings, rather than taking place in a control setup in a laboratory. The advantage of the natural setting being that it is 
more likely to yield results close to what will happen in the real world. Observational studies have rarely been conducted in the field of CIS, even though researchers have asserted that a comprehensive understanding of CIS goes beyond studying the interface's retrieval performance and the user interaction [1][14]. Conducting observational studies allowed us to have a holistic view of how collaboration happens, and hence helped in supporting the formulation of design guidelines for CCIS systems.

In our two studies, participants were given a CIS task to complete collaboratively. For each session we recorded the user screen using a screen recording tool. As Study 1 and Study 2 involved the use of existing interfaces for which we did not have the possibility to internally log user interactions, it was essential to record the participants' interactions with the interface. For this purpose we used the screen capture software Snagit $^{2}$ to record the participants' screens. The VI participants' screens were captured using a video camera, as we noticed in a pilot study that screen recording software can reduce the responsiveness of screen readers. Having to observe two collaborating participants simultaneously, taking observer notes by the researcher was very demanding. Therefore, we videotaped all co-located sessions in both study 1 and study 2, during which collaborators communicated verbally. The conversations were then transcribed by the researcher. Since the collaborators were not discussing verbally in the distributed sessions, the collaborators were not videotaped. However, Email communications and instant chat messaging logs were recorded.

As means of collecting data, video recordings and screen captures are very time consuming and labour intensive and are often prone to selective attention and researcher bias [15]. Prior to the data gathering process we predetermined measures that had to be recorded and transcribed. It was necessary to view the video recordings of each session three times, as there were a large number of measures to be recorded. These ranged from measures related to the different stages of the IS process through to measures related to the use of specific system features. Following the task, we conducted a semi-structured interview with each participant to discuss the issues encountered while performing the task. Interviews are best suited to understand people's perception of a situation and provide an opportunity for the researcher to explore people's experience in more detail [16, p.222].

The advantage of an observational study over other methods was that it allowed us to have a thorough understanding of how visually impaired and sighted users collaborated together and the challenges encountered as it gave us an insight into how the behaviour occurs in real word. Study 1 , which was considered as an exploratory study, provided us with a wealth of information about the process [17] and the behaviour patterns that occurred [10]. Nevertheless, a number of challenges occurred while making the observations and when working with the collected data. As described previously, unlike the co-located setting, in the distributed setting, only the collaborators screens were recorded but not collaborators in the sessions. Not recording these sessions left us with a number of

${ }^{2}$ https://www.techsmith.com/snagit.html unanswered questions. In some instances, the user would stop working to write down a note, as we only have the screen recording, the researcher could not know the action being performed by the user. In the majority of the cases the collaborators were close enough for the researcher to observe, therefore notetaking of the users' activities at these times was also done wherever possible.

Setting up the environment was essential for us; that is to have a view of both collaborators in order for us to interpret their interaction together and to be as unobtrusive as possible. Because the studies were mostly conducted in the VI participants workplace, we had very little control of the space. We had to position the camera in a place that both collaborators interaction can be shoot correcting and avoid to put any equipment in their way. In some sessions, the camera was not positioned correctly either due to the small room size or the organisation of the furniture. This has had a negative effect our data recording process. Additionally, as discussed for VI users, the screen recording was conducted via a camera and not a screen recording tool, because a software-based screen recording tool had a detrimental effect on the responsiveness of the screen-reader being used by the VI user. However, recording a screen with a camera can be quite problematic due to light being reflected on the screen. It was sometimes hard using a camera to capture all the interface components on the screen. This problem has been highlighted in psychology research in which inconsistency may have occurred during observations [13].

Managing the data collected during the session was extremely demanding, there were up to three video recordings for each session (the recordings for each of the 2 collaborators, and the recording of the overall session, in the case of the colocated sessions). There were a number of issues that were encountered during and after the observational sessions. These issues were related the synchronization of the videos, and the cross-modal nature of the study. Synchronizing all three recordings was essential for us to understand and interpret the conversation and the interactions. However, issues related to synchronizing arose due to two reasons. Firstly, it was hard for the researcher to turn on all three video recordings at the same time, especially in the distributed setting where the users were seated in remote locations. For these cases, we had to edit each video and cut the time difference so that effectively the recordings started from the same point in time. Secondly, due to technical issues or usability and accessibility issues encountered by the participants, the recording would occasionally have to be stopped or paused by the researcher while the issue was resolved, as it was decided that time spent resolving these issues should not be considered in the overall time of the task. This also affected the synchronization of the videos, and the researcher would have to resynchronise the videos subsequently. The cross-modal nature of the study also had an effect on the post-session interpretation of the video recording. The researcher became familiar with the synthetic voice of the screen reader and was well able to follow what the VI user was reading, even at the fast rates of speech typically used by regular screen-reader users. However, it was almost impossible to know what it was that the sighted user was looking at. This had an effect on the interpretation of the 
interactions in the session. Using tools for eye tracking can help us to identify which components of the interface are currently being viewed, but these were not available to the researcher in this study.

\section{SEMI-STRUCTURED INTERVIEWS}

Usually interview complements observational studies; as the observer will have the opportunity to ask the participants questions [18]. We also used semi-structured interviews after each observational session. The semi-structured interviews were used as a technique to gather further information in studies 1 and 2 following observation of the users performing the tasks. Semi-structured interviews provided a chance for the researcher to understand users' perceptions [16, p. 151]. The semi-structured interviews were conducted individually with each participant to complement the data collected after each task in all studies. The aim of conducting these interviews differed in each study. While in study1 the aim was to allow us to discuss in detail the issues that arose during the collaboration, in study 2 we wanted to investigate in more detail the issues encountered while using the interface, as well as the issues related to communication and collaboration. We also asked participants their views about ways to enhance this type of collaborative activity.

\section{SCENARIO-BASED INTERVIEWS}

The findings of study 1 revealed that there were clear collaboration issues that appeared in the information seeking process relating to the exploration and management stages. To further explore some of the issues related to the management of retrieved information, we interviewed seven VI and seven sighted users who participated in study 1. Scenario-based interviews allow exploration of the context of the task, along with discussions of the actions of the users. The story telling approach provides a natural way for people to describe their goals and the actions they take to achieve them in a given task context. Understanding why people do things in a specific way to achieve a goal allowed us to concentrate on the human side of the activity rather than the user interface. This allows us to explore the context, constraints, facilitators and challenges. Scenarios are adaptable and can be used at different stages in usability work. Bodker [19] discussed three ways in which scenarios could be used; to generate thoughts and ideas about a particular situation, at the starting point of a design process or to establish usability evaluation for prototypes. Scenarios allow users to envision the interaction with the interface, explain issues they encounter and recommend changes to interface components. This type of scenario narrative approach is widely used as a means of requirements gathering [20, p. 230] and helps engage participants through the use of storytelling approaches, and hence provides an understanding of the interactions people describe [21].

In the context of inclusive design and specifically designing for VI users, [22] and [23] have examined the use of scenario-based approaches in the early stages of designing prototypes. This is particularly useful if the designer is designing an interface for a multimodal or cross-modal system [23]. In this study scenarios were used to understand a situation and to identify how participants would carry out a certain tasking using the applications they normally use. The scenario facilitates participants to discuss thoughts about interacting with the applications they use, and the challenges that they encounter. When designing a scenario, we take into account the absence of the visual modality, and thus avoiding making reference to the visual aspects in graphical user interface, such as the colour or layout of the page etc. As for the VI user this type of description will not convey a useful information in the description.

This approach yielded interesting results, as it allowed VI and sighted users to envision the different scenario given to then and describe their action. This was important for us to fully understand their information management habit and the application used for such activities. This has also help us in verifying some understanding from the observational studies conducted. We have notice that participants were able to freely discuss and elaborate the actions described and go beyond the questions asked which gave us a wealth of information. It is important to assert her the role of interviewer, make the participant lead the discussion and constantly prompt the participant for feedback by asking them question related to the context. In such interviews, the participants should be more of a main 'actor' in the scenario [23].

\section{ACCESSIBILITY REVIEW}

To choose the most eligible software package for VI and sighted users to perform CCIS tasks, we determined the accessibility of possible candidate systems. The aim was not only to evaluate whether the systems complied with accessibility standards, as often websites that are claim to meet accessibility standards may still have crucial or important tasks that are difficult or even impossible to perform by the target group of users. Therefore, we aim to examine the feasibility of completing the different tasks in the candidates' interfaces using screen readers. A number of studies follow a similar evaluation approach to the one adopted here. The approach is based on looking for scenarios that bring accessibility problems to light rather than examining pages for their compliance with web accessibility standards. Mankoff et al. [24] asked a number of web-developers and VI users to perform tasks on a number of websites. By using a think-aloud protocol the participants highlighted accessibility issues they came across. Brajnik [25] developed the barrier walkthrough evaluations framework that can be used to manually evaluate the accessibility of webbased interfaces for diverse groups of users. In his approach he suggested a heuristic walkthrough [26] method based on the concept of accessibility barriers. He defines accessibility barriers as "any condition that makes it difficult to make progress or achieve an objective by a disabled person using the website through specified assistive technology" [25]. The method is an adaptation of the heuristic walkthrough [26], for usability investigations where the principles are replaced by barriers. The basic underlying idea is that, for testing and assessment purposes, it is better to start from known types of barriers rather than using general design guidelines. These barriers are derived from $\mathrm{W} 3 \mathrm{C}$ guidelines and previous accessibility investigations [25]. His approach comprised three stages: (1) defining the relevant goals and scenarios to be tested; (2) cross check the barriers with the selected pages or 
scenarios; (3) determining the severity of each barrier. The evaluation methodology conducted in our investigation was substantially based on Brajnik's [25] barrier walkthrough study.

When it comes to manually evaluating the accessibility of a web page, there is very little in the literature about the selection of evaluators [27][28]. The level of expertise has been a topic of concern in these studies [27][28]. These papers include a discussion concerning the benefits of involving an accessibility expert over involving developers with none or very little screen reader experience. Since the aim of this assessment was not only to identify accessibility barriers but to also determine if a task can effectively be completed by the target user, we decided to involve VI users in the evaluation. Involving the target users in the accessibility evaluation process can have a positive effect, as they can identify usability problems relating to task completion [29]. Two experienced VI participants were engaged with the principle researcher in performing the assessment.

The concept of barriers to accessibility was explained to the two participants. The participants were told that they would be performing an accessibility assessment exercise on the three CIS systems. The principle researcher would ask the evaluators to perform each task and then they would be asked to report any related barriers encountered during the task. Additionally, they were observed by the principal researcher while performing the tasks.

Conducting the evaluation have help us in identify the most suitable accessible interface and in identifying the issues that are needed to be tackle. It highlighted crucial accessibility and usability issues that can hinder screen reader's users from fully benefiting from a main stream CIS system. Due to time concerns in the research work done we engaged only two experienced VI participants. Both VI participants had a lot of experience using JAWS with windows, but only passing knowledge of $r$ screen reader and browsers. In such evaluation ensuring diversity and wide coverage of range of tools is important [29]. Additionally, it is important to include users with different experience level as they will also represent the target population hence having a wider coverage.

\section{USABILITY EVALUATION}

Almost any interface-driven system is likely to employ usability measurements for evaluation that involves actual users. Evaluating usability typically involves measuring ease of learning, ease of use and user satisfaction [30]. It is also common to measure things like effectiveness (typically of accomplishing the task) and efficiency (of the user doing a task). Measuring the effectiveness of the interface has been the focus of the majority of studies in the field of CIS [1]. It is evident from the literature that the purpose of performing such user studies on new CIS systems may vary. For instance, while some studies aim to identify the features users preferred [31], other studies explore the impact of the interface on the tasks performed [32]. In study 2, a number of usability inspection measures [33] were used to evaluate the users' interaction with the interface. This helped in identifying the patterns and frequencies with which the features were used, the accessibility issues encountered, and the impact these had on the process of CCIS.

For evaluating the usability, we adapted Shah and Marchionini [34] multi-modal approach for measuring usability, a usability questionnaire is used as well as analysing the data collected through the screen-recording. Data such as the frequency of use of a certain feature, number of web components access and number and type of error made were collected from screen recording. This was complimented by a post-study satisfaction questionnaire to measure the ease of use. The design of the questionnaire was influenced by the original Computer System Usability Questionnaire [35]. The questions were modified to be appropriate for the functionality of the interface and the cross-modal context of use. Responses to these questions provided information on the perceived ease of use and levels of satisfaction with the tasks.

Several previous researchers [36] [16] have performed user evaluations with pairs of participants using a CIS interface. To the best of our knowledge, previous studies in CIS has always looked at the process from one specific perspective: either the user, the collaboration, or the system. In study 2 we looked at the CIS from two points of view: the individual user interaction, by performing a usability evaluation, and the collaborative interactions, through observing the user behaviour and collaborative actions. This highlighted the effects the interface had on individual Information seeking behaviour, mutual awareness and interactions.

\section{CONCLUSION}

In this paper, we described the application of combination of qualitative and quantitative methods to achieve the goal of this project. While the quantitative measures employed enabled direct comparisons of factors such as task performance, error rates etc. between study 1 (where users employed their tools of choice) and study 2 (where users employed the modified CIS system), the qualitative measures allowed us to go deeper and explore the reasons behind the numeric results and examine less tangible but non-the-less important issues such as user satisfaction levels and how users felt about the collaboration process. Some difficulties were encountered regarding research methodology concerning the monitoring of cross-modal collaboration, particularly within distributed settings, but these did not preclude the adequate capture of results sufficient to support the analysis of individual interactions with the systems or of the collaborative process.

We believe that this paper opens an interesting discussion on ways to apply current HCI techniques in evaluating crossmodal interaction. We have reflected on the benefits and challenges of each approach used and the practical experiences we gained in applying them. These reflections are offered in the hope they may assist researchers conducting similar studies in their choice of methods. Each approach was customised to meet the needs and abilities of the user groups and the settings. In the case of the observational study, careful consideration needs to be taken when applying this approach in a crossmodal setting. As discussed, we had used a different means of data collection for each mode of interaction. /Moreover, the 
study re-enforced previous work on the value of scenario-based interviews in HCI studies involving VI users. The approach used for CIS system appraisal and selection, largely based on the barrier walkthrough method proposed by Brajnik [25], proved effective in identifying a system that was sufficiently accessible that it could, with relatively minor extensions, be employed in our second study, and so provided a platform which enabled us to infer guidelines towards the design of more accessible CCIS systems. In this respect, future work could also look into applying different evaluation techniques in cross-modal collaborative settings to determine how best to modify these protocols for use with different modes of interaction.

\section{REFERENCES}

[1] C. Shah, "Evaluating collaborative information seeking-synthesis, suggestions, and structure", Journal of Information Science, 2014.

[2] H. Hypponen, "Handbook on inclusive design of telematics applications", Stakes, 2000.

[3] F. Newell, P. Gregor, M. Morgan, G. Pullin, and C. Macaulay, "User-sensitive inclusive design," Universal Access in the Information Society, vol. 10, no. 3, pp. 235-243, 2011.

[4] K. Barnicle, "Usability testing with screen reading technology in a Windows environment," in Proceedings on the 2000 conference on Universal Usability, 2000, pp. 102-109.

[5] D. Al-Thani, "Understanding and Supporting Cross-modal Collaborative Information Seeking" PhD Thesis, School of Electronic Engineering and Computer Science, Queen Mary University of London, UK, 2016.

[6] JAWS Screen Reader. Available: http://www.freedomscientific.com/Products/Blindness/JAWS

[7] S. MacKenzie, Human-Computer Interaction: An Empirical Research Perspective. Amsterdam: Morgan Kaufmann, 2013.

[8] L. A. Suchman, Plans and situated actions: The problem of humancomputer communication. Cambridge University Press, New York, 1987.

[9] A. Blandford, " Semi-structured qualitative studies". In M. Soegaard, and R. F. Dam, Editors. The Encyclopedia of HumanComputer Interaction, the Interaction Design Foundation. 2013.

[10] D. Al-Thani, T. Stockman, and A. Tombros, "Cross-modal collaborative information seeking (CCIS): an exploratory study," in Proceedings of the 27th International BCS Human Computer Interaction Conference, 2013, p. 16-24.

[11] L. J. Skitka and E. G. Sargis, "Social psychological research and the Internet: the promise and peril of a new methodological frontier," The social net: The social psychology of the Internet, pp. $1-26,2005$.

[12] A. Dix, Human-computer interaction. Springer, 2009.

[13] S. Wilson and R. Maclean, Research Methods and Data Analysis for Psychology. McGraw-Hill Higher Education, 2011.

[14] J. Foster, Collaborative Information Behavior: User Engagement and Communication Sharing: User Engagement and Communication Sharing. IGI Global, 2010.

[15] D. Kelly, "Methods for evaluating interactive information retrieval systems with users," Foundations and Trends in Information Retrieval, vol. 3, no. 1-2, pp. 1-224, 2009.

[16] U. Flick, An Introduction to Qualitative Research, Fourth Edition edition. SAGE Publications Ltd, 2009.
[17] D. A. Althani, T. Stockman, and A. Tombros, "Exporing the Stages of Information Seeking in a cross-modal Context", Journal of Accessibility and Design for All, vol. 6, no. 1, pp. 49-80, 2016.

[18] M. Kuniavsky, Observing the user experience: a practitioner's guide to user research. Morgan kaufmann, 2003.

[19] S. Bødker, "Scenarios in user-centred design — setting the stage for reflection and action," Interacting with computers, vol. 13, no. 1, pp. 61-75, 2000.

[20] Y. Rogers, H. Sharp, and J. Preece, Interaction design: beyond human-computer interaction. Wiley, 2011.

[21] J. M. Carroll, Making use: scenario-based design of humancomputer interactions. MIT press, 2000.

[22] O. Metatla, N. Bryan-Kinns, T. Stockman, and F. Martin, "Designing for collaborative cross-modal interaction," in Proc. of Digital Engagement 2011 the 2nd RCUK Digital Economy All Hands Meeting, 2011. [1]

[23] N. G. Sahib, T. Stockman, A. Tombros, and O. Metatla, "Participatory Design with Blind Users: A Scenario-Based Approach," in Human-Computer Interaction-INTERACT 2013, Springer, 2013, pp. 685-701.

[24] J. Mankoff, H. Fait, and T. Tran, "Is your web page accessible?: a comparative study of methods for assessing web page accessibility for the blind," in Proceedings of the SIGCHI conference on Human factors in computing systems, 2005, pp. 41-50.

[25] G. Brajnik, "Web accessibility testing: when the method is the culprit," Computers Helping People with Special Needs, pp. 156163, 2006.

[26] A. Sears, "Heuristic walkthroughs: Finding the problems without the noise," International Journal of Human-Computer Interaction, vol. 9, no. 3, pp. 213-234, 1997.

[27] H. Petrie and O. Kheir, "The relationship between accessibility and usability of websites," in Proceedings of the SIGCHI conference on Human factors in computing systems, 2007, pp. 397-406.

[28] Y. Yesilada, G. Brajnik, and S. Harper, "A Barrier Walkthrough Study with Expert and Non-Expert Judges," 2009.

[29] T. Lang, "Comparing website accessibility evaluation methods and learnings from usability evaluation methods," Peak Usability, 2003.

[30] G. I. Johnson, C. W. Clegg, and S. J. Ravden, "Towards a practical method of user interface evaluation," Applied Ergonomics, vol. 20, no. 4, pp. 255-260, 1989 .

[31] S. Amershi and M. R. Morris, "CoSearch: a system for co-located collaborative web search," in Proceedings of the SIGCHI Conference on Human Factors in Computing Systems, New York, NY, USA, 2008, pp. 1647-1656.

[32] R. González-Ibáñez, M. Haseki, and C. Shah, "Let's search together, but not too close! An analysis of communication and performance in collaborative information seeking," Information Processing \& Management, vol. 49, no. 5, pp. 1165-1179, 2013.

[33] J. Nielsen, "Enhancing the explanatory power of usability heuristics," in Proceedings of the SIGCHI conference on Human Factors in Computing Systems, 1994, pp. 152-158.

[34] C. Shah and G. Marchionini, "Awareness in collaborative information seeking," Journal of the American Society for Information Science and Technology, vol. 61, no. 10, pp. 19701986,2010

[35] C. Lewis, Using the" thinking-aloud" method in cognitive interface design. IBM TJ Watson Research Center, 1982.

[36] Morris, M. R., and Horvitz, E. (2007a). SearchTogether: an interface for collaborative web search. In Proceedings of the 20th annual ACM symposium on User interface software and technology. pages 3-12, Newport, RI, USA. 